\title{
LA IMPORTANCIA DE APLICAR EL PENSAMIENTO CRÍTICO EN ENFERMERÍA
}

\author{
The importance of applying critical thinking in nursing
}

Dra. Isabel Natividad Urure Velazco ${ }^{1}$

${ }^{1}$ Decana de la Facultad de Enfermería, Universidad Nacional San Luis Gonzaga de Ica, Perú Editora en Jefe de la Revista Enfermería a la Vanguardia. Ica, Perú

Definir el pensamiento crítico es vasto, así Chaffe lo define como "el proceso activo, organizado y cognitivo, empleados para examinar cuidadosamente el propio pensamiento y el de los demás (1). Aplicado al profesional de enfermería (Alfaro-LeFevre) señala que "el pensamiento crítico se refiere al pensamiento deliberado informado, orientado a los resultados de cualquier situación y con frecuencia se usa como sinónimo de razonamiento clínico, juicio clínico, proceso enfermero, solución de problemas y toma de decisiones (2). La enfermera, que es una buena pensadora crítica, se enfrenta a los problemas, sin formar una solución rápida única, y por el contrario, se concentra en las opciones relativas a lo que puede creer y hacer (3). Esto requiere disciplina para evitar tomar decisiones prematuras. En este contexto, se puede señalar que "el pensamiento crítico es algo más que simplemente resolver problemas, más bien se trata de una mejora continua” (4).

Un elemento importante del pensamiento crítico es la creatividad, ya que es una forma de pensamiento que desemboca en el desarrollo de nuevas ideas y productos. La creatividad en la resolución de problemas y en la toma de decisiones es la capacidad para resolver y ejecutar nuevas y mejores soluciones. Se necesita creatividad cuando el profesional de enfermería se enfrenta a una situación nueva. Otro elemento importante del pensamiento crítico es la comunicación que establece la enfermera con el paciente y resto del equipo de salud, es decir que "el pensamiento crítico facilita la comunicación a través de una investigación creativa, autoconciencia y conciencia de los demás, análisis intencionado y control de los sesgos de percepción (4).

De lo expuesto, se remarca la importancia de aplicar el pensamiento crítico en enfermería, ya que "las enfermeras que han desarrollado unas buenas actividades de pensamiento crítico son las mejores comunicadoras" (4), considerando que "la comunicación es una poderosa herramienta terapéutica y una habilidad enfermera esencial utilizada para influir en los demás, y conseguir resultados de salud positivos" (4), mediante la identificación de necesidades del enfermo y ayudando en su satisfacción.

Asimismo se evidencia la importancia de aprender a pensar críticamente, porque ayuda a la enfermera a cuidar de los clientes como defensora suya y a efectuar elecciones mejor informadas sobre su cuidado. Por consiguiente, el pensamiento crítico es la clave para resolver problemas. Los profesionales de enfermería que no piensan de forma crítica se convierten en parte del problema. Los pensadores críticos se autoevalúan, autoexaminan y son capaces de mejorar por sí mismo.

Uno de los grandes problemas para la implantación de una metodología científica en el trabajo del profesional de enfermería diario es la falta de entrenamiento de la mayoría de enfermeras/os en el pensamiento crítico, analítico, el tipo de pensamiento que permite focalizar la valoración y emitir un diagnóstico de la situación en el cual sustenta el resto de las etapas del proceso de enfermería (5).

De esta forma, los profesionales de enfermería requieren actitudes y habilidades de pensamiento crítico para ejercer la profesión de forma segura competente y eficiente. 
Respecto al proceso de atención de enfermería (PAE) y el pensamiento crítico, están interrelacionados y son interdependientes. Pero son idénticos, en ambos interviene la resolución de problemas, la toma de decisiones y la creatividad. Los profesionales de Enfermería toman decisiones en el curso de resolución de problemas, como cuando se enfrenta con necesidades de diversos pacientes al mismo tiempo, deben establecer prioridades y decidir a qué paciente ayudar primero.

"Cuando la enfermera inicia un proceso enfermero, para el cuidado de un cliente específico, está también sintetizando conocimientos de pensamiento crítico, experiencia, estándares y actitudes simultáneamente" (4).

Para lograr el éxito en la sociedad actual de competitividad, se requiere de profesionales de enfermería que apliquen el pensamiento crítico o reflexivo que nos ayuda a adaptarnos a las nuevas situaciones a tomar decisiones eficaces, oportunas y competentes, con ideas creativas e innovadores en bien de la salud de los pacientes que es el centro de nuestros cuidados.

Finalizo con una interrogante:

\section{¿Aplica Usted el pensamiento crítico en enfermería que le permite mejorar cada día su actuar profesional y transformar nuestra realidad actual?}

\section{REFERENCIAS BIBLIOGRÁFICAS}

1. Chaffe J. Thinking Critically. $3^{\circ}$ ed. Boston Houghton Mifflin; 1994.

2. Alfaro - LeFevre R. Pensamiento crítico y juicio clínico en enfermería. $4^{\underline{a}}$ ed. Traducido por María Teresa Luis Rodrigo. Barcelona, España: Ed. Masson; 2009.

3. Kataoka - Yahiro M, Saylor C. A critical thinking model for nursing judgment, J. Nurs Educ. 33(8): 351). 1994.

4. Potter, Perry. Fundamentos de Enfermería. 6ํe․ Madrid, España. Ed. Grafos; 2007. Capítulo 13, p. 285-298-457-479

5. Alfaro - LeFevre R. Aplicación proceso enfermero. Barcelona, España; Ed. Masson; 2003. 